\title{
Percepção de pais acerca da inclusão social de recém-nascidos com diagnóstico de Síndrome de Down: Relato de experiência
}

Perception of parents about the social inclusion of newborns diagnosed with Down Syndrome: Experience report

Percepción de los padres sobre la inclusión social de los recién nacidos diagnosticados con Síndrome de Down: Informe de experiencia

Brenda Tanielle Dutra Barros ORCID: https://orcid.org/0000-0002-3184-050X Centro Universitário Metropolitano da Amazônia, Brasil

E-mail: brendatanielle.enf@gmail.com

Beatriz Rocha Barata de Souza ORCID: https://orcid.org/0000-0002-3796-4626 Centro Universitário Metropolitano da Amazônia, Brasil E-mail: bdbeatriz1@hotmail.com Katielem Melo Vale

ORCID: https://orcid.org/0000-0003-4868-1223 Centro Universitário Metropolitano da Amazônia, Brasil E-mail: katielem.enf@gmail.com

Rômulo Leno Miranda Barros ORCID: https://orcid.org/0000-0003-1802-4521 Universidade da Amazônia, Brasil E-mail: romuloleno@hotmail.com

Virgínia Mercês Lara Pessoa Oliveira

ORCID: https://orcid.org/0000-0002-4417-637X Centro Universitário Metropolitano da Amazônia, Brasil

E-mail: virginiaoliveira@famaz.edu.br

José Eduardo Resende Campos ORCID: https://orcid.org/0000-0002-4070-8543 Centro Universitário Metropolitano da Amazônia, Brasil E-mail: educamposjrc@gmail.com

Rubenilson Caldas Valois ORCID: https://orcid.org/0000-0001-9120-7741 Universidade do Estado do Pará, Brasil E-mail: rubenilson.valois@uepa.br

Lidiane do Socorro Carvalho dos Santos ORCID: https://orcid.org/0000-0002-6549-5986 Centro Universitário Metropolitano da Amazônia, Brasil

E-mail: lidianecarvalho90@yahoo.com.br

Joyane Mesquita Gois Andrade ORCID: https://orcid.org/0000-0001-5915-5734 Universidade da Amazônia, Brasil E-mail: joymcg17@gmail.com

Silvani Dasmasceno Barros ORCID: https://orcid.org/0000-0002-4752-6312 Centro Universitário Metropolitano da Amazônia, Brasil

E-mail: silvanedamasceno123@gmail.com

Marcely Monteiro da Silva ORCID: https://orcid.org/0000-0001-5878-5531 Centro Universitário Metropolitano da Amazônia, Brasil E-mail: marcelym98@gmail.com

Maria Barbara Freire Lameira ORCID: https://orcid.org/0000-0002-5386-7308 Centro Universitário Metropolitano da Amazônia, Brasil

E-mail: barbarafreirelameira@gmail.com

Talita Souza Moraes

ORCID: https://orcid.org/0000-0002-4776-3860 Centro Universitário do Pará, Brasil E-mail: tallitabelem@hotmail.com 


\author{
Marildete da Conceição Paula \\ ORCID: https://orcid.org/0000-0001-9092-4693 \\ Centro Universitário Metropolitano da Amazônia, Brasil \\ E-mail: marypaula72@hotmail.com \\ Tatiana Fabíola da Silva Lima \\ ORCID: https://orcid.org/0000-0001-5966-9727 \\ Universidade do Estado do Pará, Brasil \\ E-mail: tatiana.fs.lima2@gmail.com
}

\begin{abstract}
Resumo
A Síndrome de Down (SD) é uma alteração genética, portanto não é hereditária, sendo considerada a mais comum que existe. O objetivo deste estudo foi relatar uma experiência sobre a percepção de pais e/ou responsáveis acerca da inclusão social de recém-nascidos com diagnóstico de Síndrome de Down. Trata-se de um relato de experiência, realizado através de uma ação educativa, com abordagem qualitativa e descritiva, desenvolvido em uma Associação de Pais e Amigos dos Excepcionais (APAE), na Região Metropolitana de Belém, no estado do Pará, realizado no período da tarde do dia 22/10/2018. A ação educativa teve como finalidade possibilitar o esclarecimento de dúvidas e anseios de pais e/ou responsáveis legais de pessoas com SD, sobre a Trissomia do cromossomo 21 e desmistificar a discriminação no âmbito social que a criança pode sofrer, podendo facilitar a inclusão deste. Apesar das dificuldades, foi possível perceber dos pais que o nascimento de um filho com SD traz o aumento de responsabilidades, porém sua chegada é vista como um presente, e o carinho que eles recebem do filho é uma forma de compensar as dificuldades da vida diária. Percebe-se a importância eficaz da enfermagem nesse contexto, pois somos formadores de opinião, e, em essência, educadores. O profissional de saúde deve compartilhar informações sobre a saúde e os cuidados da criança com SD, para que assim seus genitores possam tomar decisões necessárias ao seu desenvolvimento.
\end{abstract}

Palavras-chave: Inclusão; Síndrome de Down; Enfermagem.

\begin{abstract}
Down Syndrome (DS) is a genetic alteration, therefore it is not hereditary, being considered the most common one that exists. The aim of this study was to report an experience about the perception of parents and / or guardians about the social inclusion of newborns diagnosed with Down syndrome. This is an experience report, carried out through an educational action, with a qualitative and descriptive approach, developed in an Association of Parents and Friends of the Exceptional (APAE), in the Metropolitan Region of Belém, in the state of Pará, carried out during the period in the afternoon of 10/22/2018. The educational action aimed to clarify the doubts and concerns of parents and / or legal guardians of people with DS, about the trisomy of chromosome 21 and demystify discrimination in the social sphere that the child may suffer, making it easier to include it. Despite the difficulties, it was possible to realize from the parents that the birth of a child with DS brings increased responsibilities, but their arrival is seen as a gift, and the affection they receive from the child is a way to compensate for the difficulties of daily life. The effective importance of nursing is perceived in this context, as we are opinion makers, and, in essence, educators. The health professional must share information about the health and care of the child with DS, so that their parents can make decisions necessary for their development.
\end{abstract}

Keywords: Inclusion; Down's syndrome; Nursing.

\title{
Resumen
}

El Síndrome de Down (SD) es una alteración genética, por lo que no es hereditario, considerándose la más común que existe. El objetivo de este estudio fue reportar una experiencia sobre la percepción de los padres y/o tutores sobre la inclusión social de los recién nacidos diagnosticados con síndrome de Down. Se trata de un relato de experiencia, realizado a través de una acción educativa, con enfoque cualitativo y descriptivo, desarrollado en una Asociación de Padres y Amigos de los Excepcionales (APAE), en la Región Metropolitana de Belém, en el estado de Pará, realizado durante el período de la tarde del 22/10/2018. La acción educativa tuvo como objetivo aclarar las dudas e inquietudes de los padres y/o tutores legales de personas con SD, sobre la trisomía del cromosoma 21 y desmitificar la discriminación en el ámbito social que pueda sufrir el niño, facilitando su inclusión. A pesar de las dificultades, fue posible darse cuenta por parte de los padres de que el nacimiento de un hijo con SD conlleva mayores responsabilidades, pero su llegada se ve como un regalo, y el cariño que reciben del niño es una forma de compensar las dificultades de la vida. la vida diaria. En este contexto se percibe la importancia efectiva de la enfermería, ya que somos formadores de opinión y, en esencia, educadoras. El profesional de la salud debe compartir información sobre la salud y los cuidados del niño con SD, para que sus padres puedan tomar las decisiones necesarias para su desarrollo. Palabras clave: Inclusión; Sindrome de Down; Enfermería.

\section{Introdução}

A síndrome de Down (SD) é considerada a cromossomopatia mais frequente e é resultante da trissomia do 
cromossomo 21, tendo como incidência 1/600 a 1/1000 nascidos vivos em todo o mundo e tendo como fator de risco a idade materna avançada (Barros et al., 2017). É conhecida como Trissomia do 21 simples, por existir um cromossomo extra ou parte de um no material genético referente ao cromossomo 21, causando triplicação ao invés de duplicação do material. Além da idade, outros fatores de risco para a síndrome está na apresentação de alterações nos cromossomos dos pais, dentre elas a SD. Por conseguinte, ela afeta o desenvolvimento da pessoa com Down e causa comprometimento intelectual, consequentemente dificuldade de aprendizagem (Silva, 2018).

As características físicas da síndrome são facilmente perceptíveis, entretanto o diagnóstico definitivo ocorre por meio da análise do cariótipo. Como em sociedade o diferente tende a ser discriminado, muitos obstáculos podem ser transpostos se as pessoas com essa condição receberem apoio de amigos, familiares e profissionais de diversas áreas, em especial os da saúde. Assim, para que o indivíduo, enquanto ser social, tenha satisfeita a necessidade desse sentir inserido em grupos, possibilitando contribuir com o desenvolvimento da pessoa com down, sendo imprescindível essa estimulação (Rodrigues \& Silva, 2015).

O conhecimento da SD pode ocorrer no período da gestação, a partir dos exames pré-natais de detecção precoce de anomalias fetais. No entanto, a maioria descobre, após o nascimento, pelos médicos majoritariamente. O diagnóstico de um filho com a Síndrome é um acontecimento traumático e desestruturante para familiares, principalmente para os pais, e que poderá alterar toda a dinâmica familiar, além de afetar o portador biopsicossocialmente. Por isso, o preparo da equipe de profissionais da saúde para o momento do diagnóstico e para o primeiro acolhimento é muito importante para potencializar o estabelecimento do vínculo da mãe com o recém-nascido (Bressan et al., 2017).

$\mathrm{O}$ preparo da equipe consiste na sensibilidade de passar o diagnóstico, porque assim como às crianças o cuidado deve ser voltado aos pais principalmente, e familiares, com a finalidade de esclarecer acerca do momento. Dessa forma, quando há na família um bebê que terá atraso no desenvolvimento, é importante que seja dado suporte formal e informal a essa família para que sejam capazes de lidar com as demandas do dia a dia de forma eficaz e para que estimulem a criança precocemente de forma satisfatória, preservando o cumprimento das necessidades individuais (Silva Filho, Gadelha \& Carvalho, 2017).

Nesse sentido, o nascimento de uma criança com a Trissomia do 21 causa impactos individuais, conjugais e parentais diferentes em pais e responsáveis, é importante que os eles possam buscar informações nas instituições especializadas, organizando-se em grupos para encontrar os melhores caminhos e informações atualizadas sobre a ocorrência genética, além de apoio e orientações necessárias para o pleno desenvolvimento de seus filhos. Essas ações contribuem para a exclusão do filho não ser a forma dos pais encararem essa realidade, dado que as crianças com Síndrome de Down podem passar grande parte de sua vida sem ter contato com outras crianças, adolescentes e adultos, e isso compromete o desenvolvimento e integração das crianças (Rabelo et al., 2017).

Para que os pais e/ou responsáveis possam dar o apoio necessário às crianças do indivíduo com a síndrome, é fundamental que estejam informados. Reis e Paula (2018), reforçam isso afirmando que:

Deve-se oferecer aos pais apoio emocional na ocasião do diagnóstico, com possibilidade de obter informações contínuas ao longo do desenvolvimento, tendo em vista os novos desafios que cada fase apresenta, além de garantir a oportunidade de trocar experiências em grupos de pais. Portanto, a oferta de serviços de apoio à família [...] tem o potencial de ampliar recursos adaptativos de enfrentamento da deficiência (Reis \& Paula, 2018, p.85).

A Inclusão Social é necessária para entender e reconhecer o outro e ter o privilégio de conviver e compartilhar com pessoas diferentes. A educação inclusiva acolhe a todos, sem exceção, e torna possível a interação com o outro. Com a inclusão, todos participam da sociedade, cada qual com suas diferenças. Destacando sobre a inclusão da pessoa com SD, é importante ressaltar que ela se inicia no âmbito familiar pela compreensão do que vem a ser a síndrome, pela aceitação da 
criança; na instituição escolar quando a criança começa a frequentá-la, pois ela se torna um dos primeiros ambientes em que a criança tem interação com outras pessoas, sendo crianças e adultos (Silva Filho, Gadelha \& Carvalho, 2017).

O grande desafio da sociedade é enxergá-los como seres humanos iguais e não apenas como alguém que tem uma limitação, que pode diminuir mediante uma ação social. Essas ações sociais podem ser realizadas em diversos setores da sociedade como educação, legislação, meio ambiente, educação, trabalho, lazer e cultura. Há necessidade de não discriminar a criança com Síndrome de Down, bem como perceber como limitadas suas capacidades intelectuais, visto que socialmente é notória a condenação da criança ao fracasso intelectual por conta da síndrome (Smeha \& Oliveira, 2014).

As pessoas com SD possuem limitações físicas e psicológicas que nem sempre as impedem de desenvolver determinadas atividades, mas que geram preconceitos individuais e coletivos devido à falta de conhecimento da população a respeito das necessidades e das características desse grupo social. Cada pessoa possui um limite a conhecer sobre si, cada um aprende de maneira diferente e ao seu tempo, e nas crianças com a síndrome não é diferente, umas se desenvolvem mais rápido que outras por terem mais estímulos, o que facilita o processo de desenvolvimento pessoal. Em vista disso, a inclusão social da criança com Síndrome de Down perpassa pela necessidade de atividades que auxiliem o desenvolvimento delas (Silva, 2018).

O objetivo deste estudo é relatar uma experiência sobre a Percepção de pais e/ou responsáveis acerca da inclusão social de recém-nascidos com diagnóstico de Síndrome de Down.

\section{Metodologia}

Trata-se de um relato de experiência, realizado através de uma ação educativa, com abordagem qualitativa e descritiva, desenvolvido em uma Associação de Pais e Amigos dos Excepcionais (APAE), na Região Metropolitana de Belém, no estado do Pará, realizado no período da tarde do dia 22/10/2018.

O relato de experiência consiste em um estudo descritivo, que traz como evidência ações que abordam uma situação vivenciada em um determinado ambiente, escolhido previamente e de interesse da comunidade científica (Flick, 2013; Lima et al., 2020).

A ação foi executada por acadêmicos do sexto período do curso de graduação em enfermagem de uma da faculdade privada, localizada na cidade de Belém, estado do Pará, com agendamento prévio no calendário da associação. O grupo foi composto por treze participantes que são ativos nas atividades ocupacionais dos filhos neste local, entretanto era estimada a presença de dezessete participantes.

Os atendimentos no local da ação são realizados durante a semana, de segunda-feira a quinta-feira, nos horários: de $8 \mathrm{~h}$ às $12 \mathrm{~h}$ e de $14 \mathrm{~h}$ às $18 \mathrm{~h}$. Nas sextas-feiras, somente atividades internas dos funcionários. A APAE realiza atendimento a pessoas com necessidade intelectual como: Microcefalia, Síndrome de Down, Autismo. Recebe apoio dos profissionais das áreas de Fisioterapia, Terapia Ocupacional, Pedagogia, Fonoaudiologia, Psicomotricidade e Assistência Social.

Preparamos o ambiente para desenvolver a dinâmica da seguinte forma: organizamos balões na parede em fileira contendo as perguntas e anexamos um banner ao lado, formamos um semicírculo com as cadeiras voltadas para a parede com os balões, para que os participantes pudessem ter contato visual e facilitar a interação entre todos. No espaço ao lado, ainda dentro do auditório, organizamos um ambiente para as crianças com materiais de pintura contendo desenhos impressos e giz de cera, para facilitar a participação dos pais na ação.

O contato, com os pais e/ou responsáveis legais, deu-se a partir do momento que os recepcionamos no local, podemos perceber que eles são dedicados no desenvolvimento de seus filhos. Observamos que é uma população de classe média e baixa, podendo ter acesso a informações ou não.

A ação educativa foi desenvolvida em duas etapas usando dez balões e um banner ilustrativo. No primeiro momento, foi aplicada uma metodologia ativa, na qual foram utilizados balões contendo perguntas abertas associadas ao tema em estudo. 
Convidamos o primeiro participante para dar início à dinâmica estourando o primeiro balão, após sua fala, a pergunta era compartilhada com os demais participantes, dando abertura à roda de conversa e assim permitindo a troca de relatos, incluindo todos os participantes.

No segundo momento, após a roda de conversa, os acadêmicos explanaram o tema através de um banner ilustrativo, contendo algumas curiosidades da Trissomia 21, dentre elas: A Síndrome de Down não é hereditária; Não existem diferentes graus de Síndrome de Down; Mesmo que eles possuam algumas semelhanças, mas não são todos iguais; Síndrome de Down não é doença e Pessoas com Síndrome de Down têm opinião! E assim, consolidando as informações para o grupo.

Quanto as questões éticas, por se tratar de um relato de experiência, onde não foi utilizado instrumentos ou outras estratégias de coletas de dados junto aos pais e/ou responsáveis, identificando-os ou permitindo a identificação exata do local no qual foi realizada a atividade, o presente relato não precisou passar pelo processo de aprovação do comitê de ética e pesquisa. A participação destes responsáveis foi voluntária, além da permissão do local para a realização desta atividade.

\section{Resultados e Discussão}

A atual sociedade é constituída por diversas pessoas, tanto em gênero como em características. Destacando -se a Síndrome de Down e a inclusão social. Muito já se evolui acerca da inclusão das pessoas com deficiência e dificuldades de aprendizagem na sociedade (Kessler \& Paschoali, 2017).

No que diz respeito às pessoas com SD, Faria (2020) alerta que a síndrome se configura como marco de desigualdade e exclusão na sociedade, fator que leva os portadores desta à dependência de benefícios sociais e da família. Observa-se que até mesmo as identidades dos familiares são modificadas pela presença de um indivíduo com SD na família e enfatiza que o estigma destas pessoas não se restringe a tais indivíduos, mas toda a família tende a ser estigmatizada.

Vale salientar que diante da sociedade temos a escolarização dessas pessoas, que é um direito de todos. Nesse ponto de vista, é que se destaca a pertinência de estudar sobre a Síndrome de Down, com enfoque especialmente não importância da família nesse processo, pois infelizmente algumas delas são as primeiras à excluí-las. Pois a cultura que vivenciamos na atualidade deve ser construída de maneira inclusiva, valorizando a diversidade e a diferença entre as pessoas, construindo o processo de interação social entre todos (Kessler \& Paschoali, 2017).

A ação educativa teve como finalidade possibilitar o esclarecimento de dúvidas e anseios de pais e/ou responsáveis legais de portadores de SD, sobre a Trissomia do cromossomo 21 e desmistificar a descriminação no âmbito social que seu filho pode correr, podendo facilitar a inclusão do seu filho na sociedade.

Durante o desenvolvimento da ação, foi notável o interesse dos pais pelos assuntos gerados, percebemos isso através do diálogo que foi desenvolvido a partir das perguntas, principalmente sobre o momento do diagnóstico e a inclusão social de seus filhos. Alguns deles já chegaram com um bom nível de esclarecimento sobre os assuntos, assim facilitando e potencializando o diálogo entre os genitores.

Observou-se que os principais profissionais atuantes no diagnóstico para estes genitores foram: Médico (Obstetra e Ginecologista), Psicólogo e Assistente Social. Identificamos que o profissional Enfermeiro foi ausente nesse momento. Inclusive, é importante destacar que no local da ação o profissional enfermeiro não se encontra presente na equipe multiprofissional.

O momento em que receberam o diagnóstico foi relatado como uma notícia surpreendente, pois em sua grande maioria, os pais foram comunicados apenas no nascimento ou meses depois, destacando a falta de preparo para receber a notícia de que o filho apresentava SD. Porém, durante a dinâmica, observou-se a resposta alguns pais, onde não desejariam ter um diagnóstico precoce e prognóstico bem-informado, pois iria gerar uma maior desestruturação emocional.

Outro fator que se soma ao momento do diagnóstico é a falta de preparo por parte dos profissionais em informar sobre 
a síndrome aos pais, devido ao modo como a informação é repassada, sendo muito objetiva e direta. Desta forma, os genitores não têm um momento específico de se prepararem para receber esta notícia.

Após o momento da descoberta da Síndrome, os responsáveis destacam que buscaram informações como uma forma de esclarecer os questionamentos que surgiam, procurando explicações em grupos de pais que passam pela mesma experiência, no ambiente familiar ou profissional da área da saúde. Segundo os pais, o resultado do esclarecimento de suas dúvidas é a diminuição das preocupações e medos que surgiam no diagnóstico, visto que não possuíam conhecimento da SD antes de terem seus filhos.

Os pais também demonstraram como é o conviver com seus filhos, discorrendo sobre os principais desafios encontrados no dia-dia. As principais queixas eram principalmente da interação com outras crianças, à lentidão na aprendizagem, inclusão escolar, inclusão social, atraso na aquisição da linguagem e no desenvolvimento motor. Dessa forma, buscam assistência com especialistas como: Geneticista, Médico (Cardiologista), Psicólogo, Fonoaudiólogo e Terapeuta Ocupacional, além do apoio da APAE, esperando que tais atrasos sejam sanados.

Por outro lado, outra temática abordada foi relacionada com as principais preocupações com o filho, em relação ao futuro. As expectativas comumente citadas são principalmente em relação à inserção na sociedade, sua independência $\mathrm{e}$ sexualidade. Desta forma, os pais buscam estimular a criança desde seu nascimento para que ela possa se desenvolver e conseguir independência em sua vida adulta.

Para os pais, a inclusão em sociedade é uma preocupação de como os seus filhos serão recebidos pelo seu comportamento e até mesmo quando estes se tornarem adultos. Também estão relacionadas aos valores pessoais dos pais e aos valores sociais que influenciam no comportamento desejável, de acordo como contexto cultural em que estão inseridos. Para atingir essas expectativas sobre o desenvolvimento dos filhos, os genitores criam algumas estratégias a fim de contribuir com a socialização (Portes, Vieira \& Faraco, 2016).

Apesar das dificuldades, foi possível perceber dos pais que o nascimento de um filho com SD traz o aumento de responsabilidades, porém sua chegada é vista como um presente, e o carinho que eles recebem do filho é uma forma de compensar as dificuldades da vida diária.

No segundo e último momento da atividade, foi explorado um banner ilustrativo abordando as principais curiosidades sobre a Síndrome. Tal ferramenta foi utilizada para consolidar as informações discutidas na roda de conversa. Dentre as curiosidades, percebemos que as mais relevantes foram a não relação com a hereditariedade, que não existem diferentes graus de Trissomia do 21, mesmo que eles possuam semelhanças, não são todos iguais, e a SD não é uma doença e que eles podem ter autonomia e independência.

\section{Conclusão}

A experiência vivenciada nesta ação foi de grande contribuição acadêmica cientifica, visto que houve estímulo, integração e participação dos pais por meio de dinâmica e abordagem explicativa. Tal interação favoreceu uma discussão entre os participantes, podendo gerar uma troca de experiências, permitindo o esclarecimento de dúvidas, proporcionando um maior conhecimento e entendimento sobre a síndrome e a inclusão social.

Esses questionamentos também demonstraram que os pais e/ou responsáveis legais tem conhecimento sobre a Trissomia do 21, sabem de seus direitos, responsabilidades e principalmente que essas crianças precisam ser estimuladas precocemente, para que elas conquistem seu lugar na sociedade.

Percebe-se a importância eficaz da enfermagem nesse contexto, pois somos formadores de opinião, e, em essência, educadores. O profissional de saúde deve compartilhar informações sobre a saúde e os cuidados da criança com SD, para que assim seus genitores possam tomar decisões necessárias para seu desenvolvimento. 


\section{Referências}

Augusti, C. \& Junqueira, L. D. M. (2016). Inclusão social para pessoas com síndrome de Down: análise de uma visita guiada pelo Congresso Nacional. Revistas UFPR. 9(3), 1-21.

Barros, A. L. O. et al. (2017). Sobrecarga de cuidadores de crianças e adolescentes com síndrome de Down. Ciência \& Saúde Coletiva [online]. 22(11), 36253634

Bressan, R. C. et al. (2017). Reverberações do atendimento em saúde na construção do vínculo mãe-bebê com síndrome de Down. Cad. Pós-Grad. Distúrb. Desenvolv. 17(2), 43-55.

Kessler, L. \& Paschoali, D. R. (2017). Considerações acerca da síndrome de Down: importância da família e o auxílio desta para o processo de inclusão. In: $8^{\circ}$ Seminário de Iniciação Científica do Curso de Pedagogia e $4^{\circ}$ Seminário Institucional Interdisciplinar PIBID, Itapiranga 2017. Anais do $8^{\circ}$ Seminário de Iniciação Científica do Curso de Pedagogia.

Faria, M. D. de. (2020). As teias que a Síndrome de Down não tece: identidade, estigma e exclusão social. Revista Crítica de Ciências Sociais, (122), 119-144.

Flick, U. Introdução à metodologia de pesquisa: um guia para iniciantes. M. Lopes (Trad.). Penso, 2013.

Lima, H. F. et al. (2020). Educação em saúde sobre doação de sangue: relato de uma experiência com crianças e adolescentes. Research, Society and Development, 9(9), e780997941.

Portes, J. R. M., Vieira, M. L. \& Faraco, A. M. X. (2016). Objetivos da socialização e estratégias de ação de pais de crianças com síndrome de Down. Act.Colom. Psicol. 19(1), 187-196.

Rabelo, G. R. G., et al. (2017). Perspectivas dos pais quanto à inclusão escolar de pessoas com síndrome de Down. ProlÍngua, 12(1), 75-85.

Reis, L. B. \& Paula, K. M. P. de. (2018). Coping materno da Síndrome de Down: identificando estressores e estratégias de enfrentamento. Estud. psicol. (Campinas), 35(1), 77-88.

Rodrigues, M. N. M. \& Silva, A. L. (2015). Impacto do nascimento de um filho com trissomia 21: perspectiva de casais idosos portugueses. Revista Pensar Enfermagem, 19(2), 54-67.

Silva, D. C. da \& Catro, O. de F. (2018). Papel do enfermeiro diante da pessoa com síndrome de Down sob a ótica do estudante de enfermagem. In: Congresso de Iniciação Científica FAPEMIG VIII, 2018, Itajubá. Anais.

Silva Filho, J. A. da, Gadelha, M. do S. N. \& Carvalho, S. M. C. (2017). Síndrome de Down: reação das mães frente à notícia e a repercussão na intervenção fisioterapêutica da criança. Revista Brasileira de Ciências da Saúde, 21(2), 157-164.

Spinazola, C. de C., et al. (2018). Correlação entre variáveis familiares: perspectiva de mães de crianças com deficiência física, síndrome de Down e autismo. Revista Educação Especial, 31(62), 697-711.

Smeha, L. N. \& Oliveira, V. L. P. de. (2014). Inclusão escolar: a perspectiva das mães de alunos com síndrome de Down. Revista Educação Especial, 27(49), 403-415. 